Serratus anterior plane block $(\mathrm{SAPB})^{5}$ could provide effective analgesia.

Methods We treated 2 female patients with PHN, unresponsive to medical therapies, 56 and 74 yo; pain was localized in the antero-lateral area of thorax, limiting free movements of ipsilateral arm. Pain was strong (NRS score 8 and 10), stabbing and burning, exacerbating in absence of stimuli and without relief during the night.

We performed an ultrasound-guided SAPB with $0.25 \%$-levobupivacaine $30 \mathrm{ml}$ and dexamethasone $8 \mathrm{mg}$ at $5^{\text {th }}$ intercostal space.

Results Both patients had immediate pain relief: NRS was 2-3 after 10 minutes, 0-1 after 2 hours. Benefit was substantially maintained over time: at 48 hours, resumption of pain was mild (NRS 2) in the first patient, moderate (NRS 4-5) in the second; in this patient we decided to perform another injection. NRS finally decreased to 2-3 and there was no need for further injections. At two months there was absence of pain for both.

Conclusions Long-lasting SAPB with local anesthetic and steroids could be a safe and effective way to treat resistant PHN, and it should be performed as soon as possible to avoid incipit of chronic pain and improve patient quality of life and outcome. Further studies are necessary to confirm our preliminary data.

\section{COMPLEX REGIONAL PAIN SYNDROME FOLLOWING POST-HERPETIC NEURALGIA}

${ }^{1} \mathrm{~A}$ Vasconcelos Pereira, ${ }^{1} \mathrm{R}$ Rodrigues Oliveira*, ${ }^{2} \mathrm{R}$ Almeida, ${ }^{2}$ J Durán, ${ }^{2} \mathrm{~A}$ Reis. ${ }^{1} \mathrm{Hospital}$ Vila Franca de Xira, Vila Franca de Xira, Portugal; ${ }^{2}$ Centro Multidisciplinar de Dor Beatriz Craveiro Lopes - Hospital Garcia de Orta, Almada, Portugal

\subsection{6/rapm-2021-ESRA.78}

Background and Aims Complex regional pain syndrome (CRPS) is a rare condition with not fully understood pathophysiology. Most cases are triggered by trauma or nerve lesion and are characterized by abnormal sensory, motor, and/or trophic findings. We present a case of CRPS following post-herpetic neuralgia, aiming to spread awareness about this poorly understood and underdiagnosed condition.

Methods A 76-year-old male with known history of hypertension and dyslipidemia was referred to the pain clinic due to uncontrolled pain in his left hand. Three months earlier, he was diagnosed with herpes zoster infection with post-herpetic neuralgia corresponding to the C6-C7 dermatome.

He complained of neuropathic pain with mechanic allodynia and hyperalgesia of the left hand, mainly in D1-D2 fingers, along with a decreased range of motion, temperature asymmetry, hypersensitivity, and diffuse edema, with significant limitation on daily activities.

Scaphotrapezoid osteoarthrosis and D1-3 edema were shown in radiography and ultrasound, respectively. Blood evaluation was unremarkable. The patient was diagnosed with CRPS by fulfilling all IASP Clinical Budapest Criteria.

Results He started multimodal therapy with gabapentin 300 $\mathrm{mg}$, progressively titrated to $300 \mathrm{mg}$ t.i.d., amitriptyline, and physiotherapy. Two months later, he reported significant functional improvement with decreasing edema and mild pain only.

Conclusions Varicella-zoster virus (VZV) is a known precipitating factor in CRPS. However, it is still unknown whether the disease occurs as a continuum of post-herpetic neuralgia or as a consequence of VZV neurotropism. Pharmacotherapy with gabapentinoids and tricyclic antidepressants have an important role in symptom control, but an early diagnosis and prompt physical rehabilitation are the cornerstones for successful outcomes.

\section{THORACIC PARAVERTEBRAL BLOCK FOR CHRONIC PAIN CAUSED BY THORACIC PARAVERTEBRAL MASS IN LEUKEMIA PATIENT}

A Ghidan*, H Abu Khudair, E Farhoud. King Hussein Cancer Center, Amman, Jordan

\subsection{6/rapm-2021-ESRA.79}

Background and Aims Thoracic paravertebral is now an established regional anesthetic technique for unilateral surgical procedures or painful conditions. It has fewer complications when performed correctly.

Chronic pain is a significant concern in a cancer patient. Morphine has been used as a first-line analgesic strategy for severe pain. The effect of morphine on tumour growth is still contradictory, as both growth-promoting and growth-inhibiting effects have been observed. Accumulating evidence suggests that morphine can affect the proliferation and migration of tumour cells as well as angiogenesis (1)

Methods We report here the use of Ultrasound-guided right side thoracic paravertebral block at the level of T2 and T9 for severe back pain, right side chest wall and right side abdominal wall pain of T2 and T9 dermatomes in 56 years old male patient, known case of acute myeloid leukemia (AML-M2) found to have right side thoracic paravertebral masses at levels of T2 and T9.

The 19-gage Tuohy needle inserted USG In-plane, after penetration of internal intercostal membrane $0.25 \%$ bupivacaine $20 \mathrm{ml}$ with dexamethasone $4 \mathrm{mg}$ was injected after negative aspiration of blood or air. Total amount: $40 \mathrm{ml}$ of $0.25 \%$ Marcaine $(20 \mathrm{ml}$ each injection) with Dexamethasone total of $8 \mathrm{mg}$.

Results During the block procedure, no difficulty of breath or desaturation occurred and no hemodynamic change occurred.

In the PACU, the patient was comfortable and free of pain then discharged home. He remains pain-free for 2 days.

Conclusions Thoracic paravertebral block is an alternative of morphine for chronic pain caused by thoracic paravertebral mass.

\section{$80 \quad$ METHADONE IN PAIN MANAGEMENT}

H Senderovich*. Baycrest, University of Toronto, Toronto, Canada

\subsection{6/rapm-2021-ESRA.80}

Background and Aims Adequate analgesia can be challenging, as pharmacological options are not necessarily effective for all types of pain and have various side effects. Methadone is increasingly being considered in the management of both cancer- and non-cancer-related pain.

Objective: To summarize the evidence on the effectiveness of methadone and review the side effects and cost of this drug.

Methods PubMed, Medline, Embase, and Google Scholar databases were searched to identify Randomized Controlled Trials (RCTs) assessing methadone and a comparison drug. 